\title{
Oxidative phosphorylation efficiency, proton conductance and reactive oxygen species production of liver mitochondria correlates with body mass in frogs
}

\author{
Damien Roussel ${ }^{1, *}$, Karine Salin ${ }^{1,2}$, Adeline Dumet ${ }^{1}$, Caroline Romestaing ${ }^{1}$, Benjamin Rey ${ }^{3,4}$ and Yann Voituron ${ }^{1}$
}

\begin{abstract}
Body size is a central biological parameter affecting most biological processes (especially energetics) and the mitochondrion is a key organelle controlling metabolism and is also the cell's main source of chemical energy. However, the link between body size and mitochondrial function is still unclear, especially in ectotherms. In this study, we investigated several parameters of mitochondrial bioenergetics in the liver of three closely related species of frog (the common frog Rana temporaria, the marsh frog Pelophylax ridibundus and the bull frog Lithobates catesbeiana). These particular species were chosen because of their differences in adult body mass. We found that mitochondrial coupling efficiency was markedly increased with animal size, which led to a higher ATP production (+70\%) in the larger frogs ( $L$. catesbeiana) compared with the smaller frogs ( $R$. temporaria). This was essentially driven by a strong negative dependence of mitochondrial proton conductance on body mass. Liver mitochondria from the larger frogs ( $L$. catesbeiana) displayed $50 \%$ of the proton conductance of mitochondria from the smaller frogs ( $R$. temporaria). Contrary to our prediction, the low mitochondrial proton conductance measured in L. catesbeiana was not associated with higher reactive oxygen species production. Instead, liver mitochondria from the larger individuals produced significantly lower levels of radical oxygen species than those from the smaller frogs. Collectively, the data show that key bioenergetics parameters of mitochondria (proton leak, ATP production efficiency and radical oxygen species production) are correlated with body mass in frogs. This research expands our understanding of the relationship between mitochondrial function and the evolution of allometric scaling in ectotherms.
\end{abstract}

KEY WORDS: Allometry, Bioenergetics, Mitochondrial efficiency, Free radicals, Proton leak

\section{INTRODUCTION}

Physiological traits including longevity, fecundity, and endurance to starvation and desiccation have previously been shown to be closely related to body size, placing it as one of the most important parameters in an animal's life history (Schmidt-Nielsen, 1984). As energy is essential for all biological activity, the link between body size and energy metabolism has previously been extensively studied

${ }^{1}$ Laboratoire d'Ecologie des Hydrosystèmes Naturels et Anthropisés, UMR 5023, CNRS, Université de Lyon 1, 69622 Villeurbanne, France. ${ }^{2}$ Institute of Biodiversity, Animal Health and Comparative Medicine, University of Glasgow G12 8QQ,

Glasgow, UK. ${ }^{3}$ Laboratoire de Biométrie et Biologie Évolutive, UMR 5558, CNRS, Université de Lyon 1, 69622 Villeurbanne, France. ${ }^{4}$ Brain Function Research Group, School of Physiology, Faculty of Health Sciences, University of the Witwatersrand, Johannesburg 2193, South Africa.

*Author for correspondence (damien.roussel@univ-lyon1.fr)

Received 28 May 2015; Accepted 18 August 2015
(Schmidt-Nielsen, 1984; Darveau et al., 2002; Glazier, 2005). Most notably, basal metabolic rate in mammals and birds has been correlated with many energy-consuming processes at the level of tissues, cells and mitochondria (Kunkel and Campbell, 1952; Hulbert and Else, 2000; Else et al., 2004), providing support for the 'multiple-causes model' of allometry (Darveau et al., 2002). Research has focused on the relationship between body mass $\left(M_{\mathrm{b}}\right)$ and mitochondrial function (Darveau et al., 2002; Brand et al., 2003; Porter and Brand, 1993). Understanding the link between body size and mitochondrial bioenergetics is of fundamental importance as mitochondria are essential organelles of eukaryotic cells responsible for the biosynthesis of many cellular metabolites and the generation of chemical energy in the form of ATP (Brand, 2005).

Allometric relationships of proton leak and oxidative activity with animal body size have been observed in the liver mitochondria of endotherms (Brand et al., 2003; Porter and Brand, 1993; Porter et al., 1996; Polymeropoulos et al., 2012). Proton leak refers to the mechanism where motive force is dissipated through the mitochondrial inner membrane proton conductance pathways independently of ATP synthase. In turn, proton leak diverts energy away from ATP production, resulting in the inefficiency of mitochondria. Therefore, previous studies suggest that small endotherms have a lower mitochondrial efficiency than larger species (Brand et al., 2003; Porter and Brand, 1993; Porter et al., 1996). These allometric relationships echo several experimental studies on (i) organisms treated with chemical uncouplers such as 2,4-dinitrophenol (Toyomizu et al., 1992; Caldeira da Silva et al., 2008; Salin et al., 2012a) or (ii) a genetic line of poultry selected for low feed efficiency (Bottje et al., 1999), showing that mitochondrial efficiency could constrain growth rate and so underpin the variability of $M_{\mathrm{b}}$. However, the question of why mitochondrial inefficiency evolved in an allometric manner among taxa remains an open debate. One possible explanation is that mitochondrial efficiency is constrained by the generation of reactive oxygen species (ROS). ROS are the by-product of normal mitochondrial activity and play an important role in the rate of ageing and lifespan. According to the 'uncoupling to survive' theory, stimulating proton leak would lower mitochondrial efficiency in addition to the generation of ROS and this would result in an advantage to long-lived species (Brand, 2000).

However, there are not as many studies on ectotherms. In terrestrial ectotherms, respiratory metabolic rate correlates with $M_{\mathrm{b}}$ (Nagy, 2005), but this is less clear at the cellular and mitochondrial levels (Brookes et al., 1998; Else and Hulbert, 1985; Hulbert et al., 2002). However, studies investigating the relationship between mitochondrial activity and $M_{\mathrm{b}}$ in ectotherms are scarce. Studies available showing no relationship may simply reflect the disparate nature of the ectotherms studied or experimental procedures used as discussed in Hulbert et al. (2002). The aim of the present study was to investigate whether parameters of mitochondrial bioenergetics, 
Table 1. Body mass, liver mass and mitochondrial respiratory properties of different frog species

\begin{tabular}{|c|c|c|c|c|c|}
\hline & R. temporaria & P. ridibundus & L. catesbeiana & Mass effect & Species effect \\
\hline$M_{\mathrm{b}}(\mathrm{g})$ & $18 \pm 1$ & $47 \pm 4$ & $239 \pm 14$ & - & $F=320.1, P<0.001^{* *}$ \\
\hline Liver mass (g) & $0.48 \pm 0.06$ & $1.18 \pm 0.21$ & $5.06 \pm 0.47$ & $F=12.5, P=0.001^{* *}$ & n.s. \\
\hline $\begin{array}{l}\text { Liver mass }\left(\mathrm{g} 100 \mathrm{~g}^{-1} M_{\mathrm{b}}\right) \\
\dot{V}_{\mathrm{O}_{2}}\left(\mathrm{nmol} \mathrm{O} \min ^{-1} \mathrm{mg}^{-1} \text { protein }\right)\end{array}$ & $2.7 \pm 0.2$ & $2.5 \pm 0.2$ & $2.2 \pm 0.1$ & $F=17.4, P=0.0002^{* *}$ & $F=7.7, P=0.002^{* *}$ \\
\hline State $3 \mathrm{ADP}$ & $7.8 \pm 0.6$ & $8.8 \pm 1.3$ & $7.7 \pm 1.0$ & n.s. & n.s. \\
\hline State 4 oligo & $2.7 \pm 0.2$ & $2.8 \pm 0.2$ & $2.2 \pm 0.2$ & $F=5.63, P=0.02^{*}$ & n.s. \\
\hline State $3_{\mathrm{FCCP}}$ & $10.0 \pm 0.9$ & $11.6 \pm 1.6$ & $12.3 \pm 1.8$ & $F=8.61, P=0.007^{* *}$ & $F=6.12, P=0.006^{* *}$ \\
\hline \multicolumn{6}{|l|}{ Respiratory parameters } \\
\hline RCR & $3.1 \pm 0.2$ & $3.0 \pm 0.3$ & $3.4 \pm 0.2$ & $F=7.3, P=0.01^{* *}$ & $F=4.41, P=0.02^{*}$ \\
\hline $\mathrm{fAS}_{\mathrm{m}}$ & $3.9 \pm 0.3$ & $4.1 \pm 0.4$ & $5.4 \pm 0.4$ & $F=10.46, P=0.003^{* *}$ & $F=6.12, P=0.006^{* *}$ \\
\hline
\end{tabular}

Values are expressed as means \pm s.e.m. The number of independent mitochondrial preparations was: Lithobates catesbeiana (bull frog), $N=10 ; P e l o p h y l a x$ ridibundus (marsh frog), $N=10$; and Rana temporaria (common frog), $N=17$. Effects were considered significant for $P<0.05$ ( $P<0.05$; ${ }^{* *} P<0.01$ ).

$M_{\mathrm{b}}$, body mass; $\dot{V}_{\mathrm{O}_{2}}$, rate of oxygen consumption; state $3_{\mathrm{ADP}}$, ADP-stimulated respiration; state 4 oligo, basal non-phosphorylating respiration measured in the presence of $5 \mu \mathrm{g} \mathrm{ml}^{-1}$ oligomycin; state $3_{\mathrm{FCCP}}$, FCCP-induced maximal respiration measured in the presence of $2 \mu \mathrm{mol} \mathrm{I}^{-1} \mathrm{FCCP}$; RCR, respiratory control ratio, calculated as the ratio of state $3_{\mathrm{ADP}}$ to state $4_{\text {oligo }}$; $\mathrm{fAS}_{\mathrm{m}}$, fractional mitochondrial aerobic scope, calculated as the ratio of state $3_{\mathrm{FCCP}}$ to state $4_{\text {oligo }}$.

including ATP synthesis efficiency, proton conductance and ROS generation, show an allometric relationship with $M_{\mathrm{b}}$ in frog species from the same family [Ranidae: Rana temporaria L., Pelophylax ridibundus (Pallas 1771) and Lithobates catesbeiana (Shaw 1802)]. Our results show a strong negative correlation between proton conductance and $M_{\mathrm{b}}$, and a positive correlation between mitochondrial ATP synthesis efficiency and $M_{\mathrm{b}}$. Interestingly, the larger frog species, which display high mitochondrial ATP synthesis efficiency and low inner membrane proton conductance, also exhibit a low level of mitochondrial ROS production.

\section{RESULTS}

\section{$M_{\mathrm{b}}$ and liver mass}

$M_{\mathrm{b}}$ and liver mass of each of the frog species are listed in Table 1. The liver represented a smaller proportion of total $M_{\mathrm{b}}$ as the size of the frog species increased (Table 1). This trend resulted in an allometric relationship of liver mass to $M_{\mathrm{b}}$ with an exponent of 0.95 (Fig. 1).

\section{Mitochondrial oxidative phosphorylation activity}

Table 1 shows the respiratory parameters of liver mitochondria isolated from each of the frog species. The mitochondrial oxygen consumption measured at the maximum rate of ATP synthesis

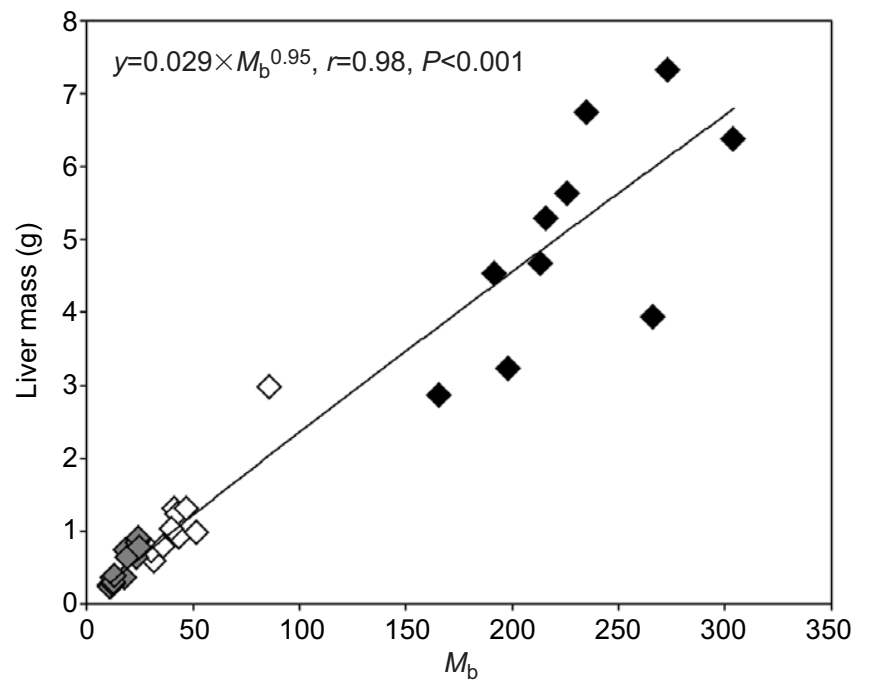

Fig. 1. Allometric relationship between liver mass and body mass $\left(\boldsymbol{M}_{\mathrm{b}}\right)$ of different-sized frogs. The line is the best power fit to the data as described by the equations. Lithobates catesbeiana (bull frog), black; Pelophylax ridibundus (marsh frog), white; and Rana temporaria (common frog), grey. (state 3) was not significantly different between the three species, or significantly affected by $M_{\mathrm{b}}$. In contrast, the rate of basal nonphosphorylating oxygen consumption, measured in the presence of oligomycin (state $4_{\text {oligo }}$ ), and the rate of uncoupled oxygen consumption, measured in the presence of FCCP (state 3 FCCP), were both significantly correlated to $M_{\mathrm{b}}$. The respiratory control ratios (RCRs) were significantly different between species and were significantly dependent on $M_{\mathrm{b}}$ (Table 1$)$. Importantly, mean RCR values obtained $(3.2 \pm 0.1)$ in this study fall within the range of values previously published for ectotherm liver mitochondria respiring on succinate (1.4 to 5.8; Akhmerov, 1986; Brand et al., 1991; Hulbert et al., 2002; Savina et al., 2006; Salin et al., 2012b). Calculated fractional mitochondrial aerobic scope $\left(\mathrm{fAS}_{\mathrm{m}}\right)$ was significantly higher in larger individuals (Table 1), with a significant dependence on $M_{\mathrm{b}}\left(\mathrm{fAS}_{\mathrm{m}}=2.4 \times M_{\mathrm{b}}{ }^{0.14}\right.$, where $M_{\mathrm{b}}$ is in $\left.\mathrm{g} ; R^{2}=0.28, P<0.001\right)$. These data are in line with previously published data in birds with an exponent of 0.09 (Brand et al., 2003) or in mammals with an exponent of 0.12 (Porter and Brand, 1993; Porter et al., 1996) and suggest that larger species have higher effective mitochondrial electron transport capacity than smaller species.

\section{Mitochondrial efficiency}

Fig. 2A shows the linear relationship between the rates of ATP synthesis and oxygen consumption measured in the presence of an ATP-regenerating system (hexokinase plus glucose) in liver mitochondria isolated from each of the frog species. As for the respiratory parameters reported above, the mean maximal rates of ATP synthesis and oxygen consumption (highest points to the right of the linear relationships) were not significantly different between species. The slopes of the linear relationships were positively mass dependent $\left(\mathrm{ATP} / \mathrm{O}=0.4 \times M_{\mathrm{b}}{ }^{0.23}, R^{2}=0.23, P=0.017\right)$, indicating that the larger frogs had a higher mitochondrial coupling efficiency than those with a smaller $M_{\mathrm{b}}$ (Fig. 2B). This is further highlighted by the fact that, when calculated at the highest common oxygen consumption rate $\left(7.3 \mathrm{nmol} \mathrm{O} \mathrm{min}^{-1} \mathrm{mg}^{-1}\right)$, liver mitochondrial ATP production increased as a function of $M_{\mathrm{b}}$ [ATP synthesis (nmol ATP $\left.\min ^{-1} \mathrm{mg}^{-1}\right)=1.2 \times M_{\mathrm{b}}{ }^{0.33}, R^{2}=0.29, P=0.0006$; mass effect: $F=4.4, \quad P=0.04$; species effect: $F=2.6, P=0.08$; mass $\times$ species: $F=2.5, \quad P=0.1]$, with mitochondria from the largest frog (L. catesbeiana) producing on average $70 \%$ more ATP than mitochondria from the smallest frog ( $R$. temporaria).

\section{Mitochondrial membrane potential and proton conductance}

The values of proton-motive force $(\Delta p)$ and its chemical $(\Delta \mathrm{pH})$ and electrical (mitochondrial membrane potential, $\Delta \psi$ ) components 

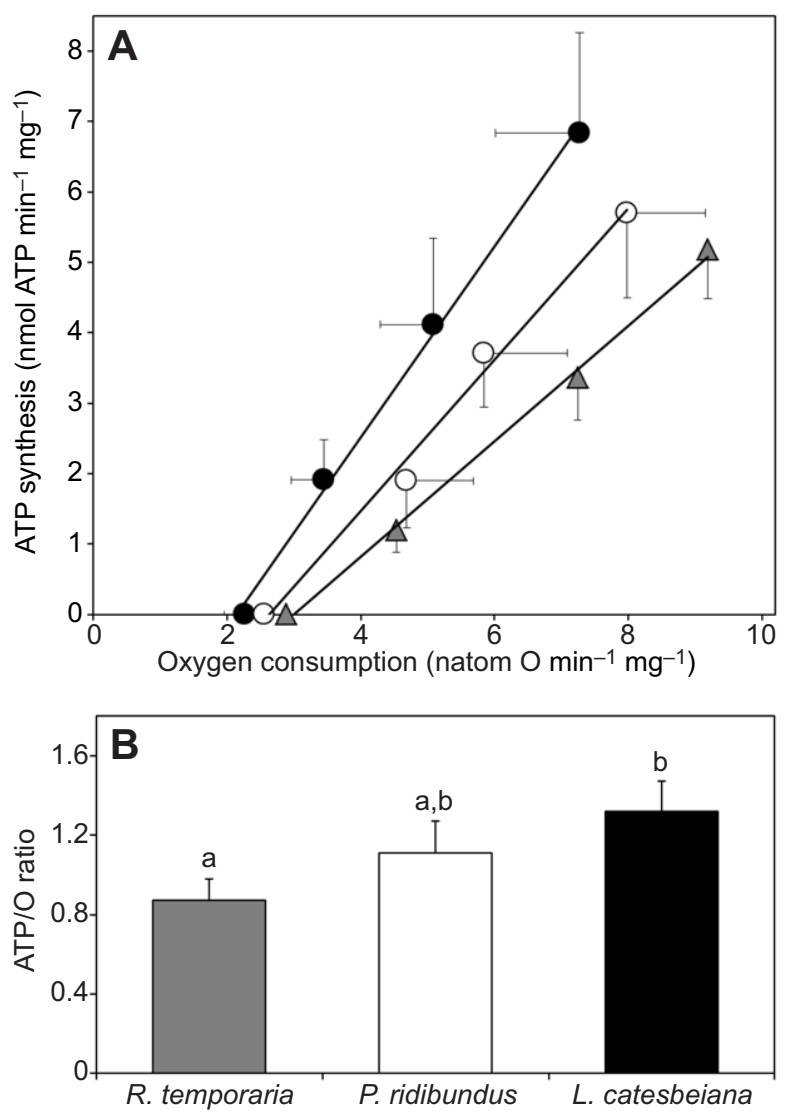

Fig. 2. Mitochondrial oxidative phosphorylation efficiency in different frog species. (A) Relationship between ATP synthesis and oxygen consumption in liver mitochondria from bull frog (L. catesbeiana; black), marsh frog ( $P$. ridibundus; white) and common frog ( $R$. temporaria; grey). Values are means \pm s.e.m. of $N=9$ (bull frog), $N=10$ (marsh frog) and $N=17$ (common frog) independent mitochondrial preparations. (B) Mitochondrial efficiency (ATP/O), calculated as the slope of the linear relationship curves presented in A. Data with different superscript letters are significantly different at $P<0.05$.

were determined in frog mitochondria respiring on succinate under basal non-phosphorylating steady-state conditions (state $4_{\text {oligo }}$ ). Table 2 shows that the values of $\Delta p, \Delta \mathrm{pH}$ and $\Delta \psi$ in L. catesbeiana were significantly higher than values in $R$. temporaria. Fig. 3 reports the response of substrate oxidation and proton leak systems to mitochondrial membrane potential. The response of substrate oxidation rate was determined by manipulating the mitochondrial membrane potential with an increasing dose of FCCP, a known uncoupler (Brand and Nicholls, 2011). Fig. 3A shows that the liver substrate oxidation system of $R$. temporaria mitochondria was less active than that of mitochondria from the other two species, as it consumed oxygen more slowly at any given value of mitochondrial

Table 2. Characteristics of proton-motive force in different frog species

\begin{tabular}{lccc}
\hline & R. temporaria & P. ridibundus & L. catesbeiana \\
\hline$\Delta \psi(\mathrm{mV})$ & $141 \pm 3^{\mathrm{a}}$ & $158 \pm 4^{\mathrm{b}}$ & $160 \pm 3^{\mathrm{b}}$ \\
$\Delta \mathrm{pH}(\mathrm{mV})$ & $9 \pm 1^{\mathrm{a}}$ & $11 \pm 1^{\mathrm{a}, \mathrm{b}}$ & $13 \pm 1^{\mathrm{b}}$ \\
$\Delta p(\mathrm{mV})$ & $150 \pm 3^{\mathrm{a}}$ & $169 \pm 4^{\mathrm{b}}$ & $173 \pm 2^{\mathrm{b}}$ \\
\hline
\end{tabular}

Electrical $(\Delta \psi)$ and chemical $(\Delta \mathrm{pH})$ components of mitochondrial proton-motive force $(\Delta p)$ were measured in frog mitochondria respiring on succinate in the presence of rotenone and oligomycin (state $4_{\text {oligo }}$ ). See Materials and methods for details.

Values are means \pm s.e.m. of $N=6$ ( $L$. catesbeiana and $P$. ridibundus) and $N=13$ (R. temporaria) independent mitochondrial preparations. Data with different superscript letters are significantly different at $P<0.05$.
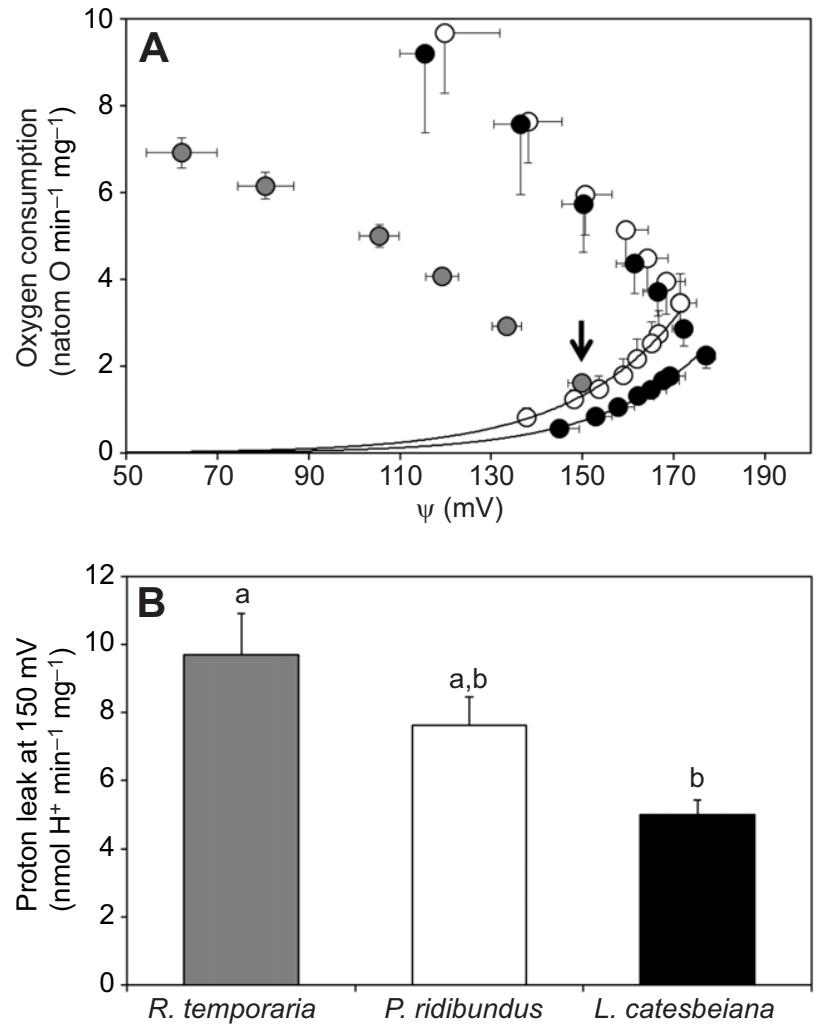

Fig. 3. Mitochondrial proton leak activity in different frog species. (A) Kinetics of proton leak in liver mitochondria isolated from bull frog ( $L$. catesbeiana; black), marsh frog ( $P$. ridibundus; white) and common frog (R. temporaria; grey). $\psi$, mitochondrial membrane potential. (B) Proton conductance was calculated from non-phosphorylating respiration rate at $150 \mathrm{mV}$ (arrow in A), assuming a constant stoichiometry of $6 \mathrm{H}^{+} / \mathrm{O}$ for oxidation of succinate for $L$. catesbeiana, $P$. ridibundus and $R$. temporaria. See Materials and methods for details. Values are means \pm s.e.m. of $N=6$ (bull frog and marsh frog) and $N=13$ (common frog) independent mitochondrial preparations. Data with different superscript letters are significantly different at $P<0.05$.

membrane potential. Mitochondrial proton leak at $150 \mathrm{mV}$, the highest common value of membrane potential (Fig. 3A), was significantly different between species $(F=9.7, P=0.001$; Fig. 3B), with mitochondria from the larger frog (L. catesbeiana) having only $50 \%$ of the proton conductance of liver mitochondria from the smaller frog ( $R$. temporaria) on average. The proton leak rate at $150 \mathrm{mV}$ was also negatively dependent upon mass $(F=13.4$, $P=0.001$ ) as shown in Fig. 4A.

\section{Mitochondrial ROS production and superoxide dismutase activity}

ROS production showed a negative correlation with $M_{\mathrm{b}}$ (mass effect: $F=9.3, P=0.005)$, but not between species $(F=1.6, P=0.22)$. Interestingly, the interaction between these two factors was significant (mass $\times$ species: $F=3.8, P=0.03$ ) suggesting that the effect of $M_{\mathrm{b}}$ on ROS production is greater in $R$. temporaria than in $P$. ridibundus and $L$. catesbeiana. Therefore, liver mitochondria from the larger individuals produced significantly lower oxygen radical (per mg protein) than those from the smaller animals (Fig. 4B). When ROS production is estimated from the efflux of hydrogen peroxide, as here, it is assumed that the activity of superoxide dismutase is not limiting and not different between species. The activity of liver superoxide dismutase was not significantly different between frogs $\left(21.0 \pm 0.9 \mathrm{U} \mathrm{mg}^{-1}\right.$ protein in 

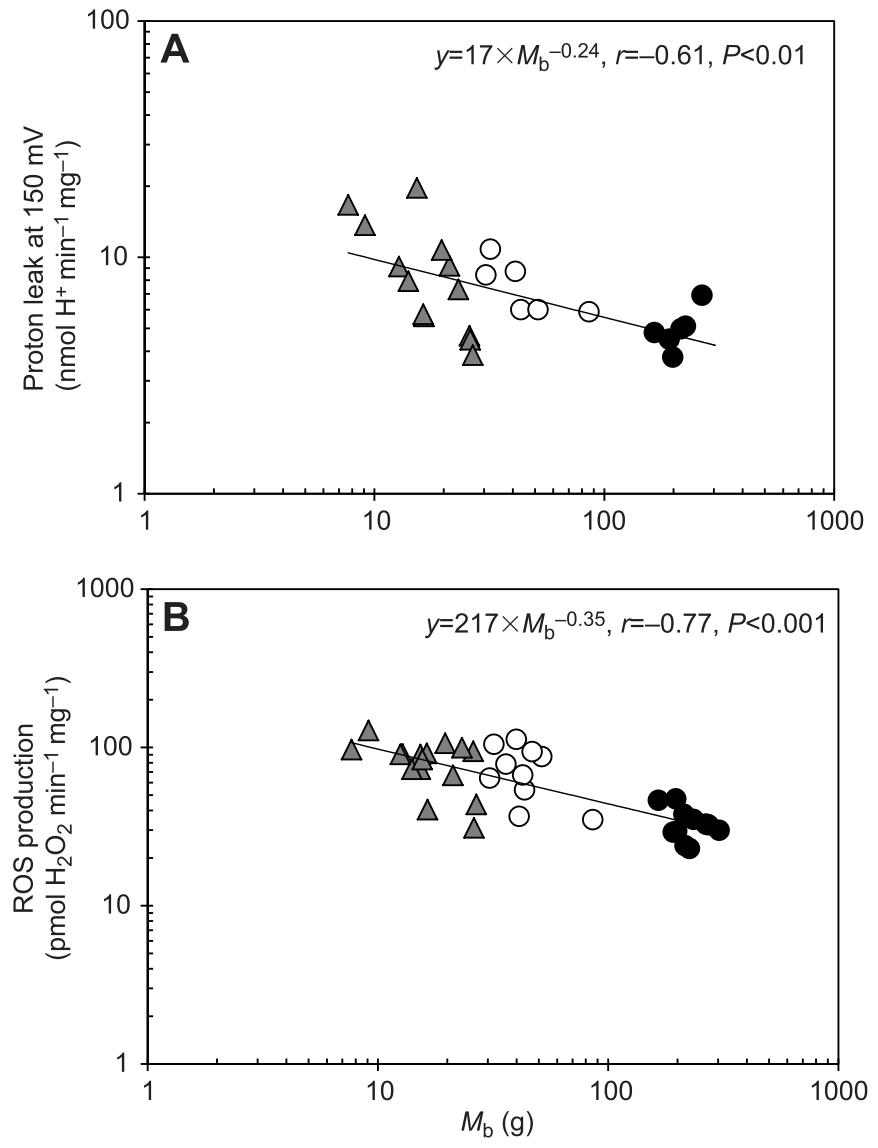

Fig. 4. Allometric relationship between mitochondrial proton leak rate at $150 \mathrm{mV}$ or mitochondrial reactive oxygen species production and $\boldsymbol{M}_{\mathrm{b}}$ in frogs. (A) Proton leak rate and (B) reactive oxygen species (ROS) production are plotted against $M_{\mathrm{b}}$ for bull frog $(L$. catesbeiana; black, $N=6-11)$, marsh frog ( $P$. ridibundus; white, $N=6-10)$ and common frog ( $R$. temporaria; grey,

$N=13-16)$. $N$ refers to the number of independent mitochondrial preparations. See Materials and methods for details. The lines are best power fits to the data as described by the equations.

$R$ temporaria; $21.4 \pm 4.5 \mathrm{U} \mathrm{mg}^{-1}$ protein in P. ridibundus; $20.7 \pm$ $2.3 \mathrm{U} \mathrm{mg}^{-1}$ protein in L. catesbeiana). Therefore, the differences in hydrogen peroxide efflux rate between frog species would not be explained by differences in superoxide dismutase activity.

\section{DISCUSSION}

The mitochondrial coupling ATP/O ratio is of physiological and ecological importance as it shows how much oxygen and nutrients are needed to produce ATP, the main form of energy usable by cells. The results in this study indicate that mitochondria from larger frogs produce more ATP per amount of oxygen consumed and therefore per amount of nutrients oxidized than smaller frogs. This suggests that larger frogs have a lower cost of cellular functioning, as their mitochondria are set to minimize the cost of ATP production and therefore optimize energy output for cellular processes. Such a difference in mitochondrial coupling efficiency has previously been shown to correlate with intraspecific variation in $M_{\mathrm{b}}$ in natural populations of common frogs (Salin et al., 2012b). It has been suggested that a change in mitochondrial membrane proton leak is one of the underlying biochemical mechanisms involved in the variability of mitochondrial efficiency in common frogs (Salin et al., 2012b). To support this, we have found that there is a strong negative correlation between proton conductance and $M_{\mathrm{b}}$, similar to previous studies on avian and mammalian liver mitochondria
(Porter and Brand, 1993; Brand et al., 2003). However, this result contradicts previous studies that have shown no allometric relationship between mitochondrial proton leak and $M_{\mathrm{b}}$ in ectotherms (Hulbert et al., 2002). The differences in results between studies may simply reflect the disparate nature of the ectotherm species used in each study (fishes, toads, lizards, crocodiles, snails) in addition to the temperature and tissue studied (kidney, liver, skeletal muscle). Although the data were collected from a small number of frog species, they suggest that the negative relationship between proton conductance and $M_{\mathrm{b}}$ that is seen in endotherms (Porter and Brand, 1993; Porter et al., 1996; Polymeropoulos et al., 2012) may also occur in frogs. However, this may not necessarily be the case for all groups of ectotherms (Hulbert et al., 2002), as suggested in reptiles, where proton conductance of liver mitochondria from crocodiles appears greater than that of smaller reptiles (Brookes et al., 1998; Hulbert et al., 2002). The results in this study show that under resting conditions, liver mitochondria from larger frogs have lower costs of energy maintenance, consuming less oxygen to balance proton leak across the inner membrane.

The lower mitochondrial proton permeability found in the larger species might be explained by at least three mechanisms: decreased surface area per mass of protein, changes in mitochondrial phospholipid fatty acyl composition and/or changes in some specific membrane proteins. As an example, the surface area of liver mitochondria decreases as $M_{\mathrm{b}}$ increases in mammals (Else and Hulbert, 1985; Porter et al., 1996), accounting for almost $70 \%$ of the differences in proton leak (Porter et al., 1996). However, this does not seem to be the case in liver mitochondria from reptiles (Else and Hulbert, 1985) or birds (Else et al., 2004), showing that other characteristics of the inner membrane are required to explain the relationship between $M_{\mathrm{b}}$ and proton leak in these vertebrates. Another potential mechanism involves the fatty acid composition of the mitochondrial membrane phospholipids. Previous research has shown that the proton conductance of the native mitochondrial membrane positively correlates with the polyunsaturated fatty acid content of phospholipids (Porter et al., 1996; Brookes et al., 1998; Else et al., 2004). However, the loss of the phylogenetic and allometric differences in proton conductance found with liposomes made from the mitochondrial membrane (Brookes et al., 1997) further highlights that the presence of mitochondrial membrane proteins plays an important role in determining proton permeability (Stuart et al., 2001). For example, this has been suggested for the content of adenine nucleotide translocase (Talbot et al., 2004; Brand et al., 2005; Shabalina et al., 2006). In amphibians, some studies have shown that the mitochondrial inner membrane surface area might be greater in the mitochondria of liver and muscle from smaller species (Brookes et al., 1998; Hulbert et al., 2006; Berner et al., 2009). Therefore, the relationship between $M_{\mathrm{b}}$ and proton conductance in frogs might be due to variation in the mitochondrial inner membrane. This could be caused by changes in the inner membrane fatty acyl composition and/or in the inner membrane protein content (such as adenine nucleotide translocase isoforms); however, this remains to be clarified and requires future investigation.

Several studies have linked ROS production with proton-motive force (Korshumov et al., 1997; Miwa et al., 2003; Kikusato and Toyomizu, 2013). In this context, mitochondria with lower basal proton conductance would show a high membrane potential and greater ROS production. To investigate this further, we measured succinate-driven ROS production in the liver mitochondria, a process that occurs during reverse electron transport and is therefore 
critically sensitive to proton-motive force and mild uncoupling (Korshumov et al., 1997; Miwa et al., 2003; Mookerjee et al., 2010). We found that liver mitochondria from the larger individuals produced significantly lower levels of ROS (per mg protein) than those from the smaller animals. A similar negative relationship between $M_{\mathrm{b}}$ and ROS production can be found in the literature for liver, kidney, heart and fibroblasts in mammals (Sohal et al., 1989, 1990; Ku et al., 1993; Csiszar et al., 2012). However, such interspecific correlation between mammalian $M_{\mathrm{b}}$ and mitochondrial ROS generation is not systematically found, being mostly revealed when isolated mitochondria oxidize succinate in the absence of rotenone (Lambert et al., 2007) or when cells are metabolically stressed (Csiszar et al., 2012). However, it is apparent that the negative relationship reported between $M_{\mathrm{b}}$ and proton leak (Porter and Brand, 1993; Porter et al., 1996; Polymeropoulos et al., 2012) cannot be associated with a positive allometric variation in ROS generation (Sohal et al., 1989, 1990; Ku et al., 1993; Lambert et al., 2007; Csiszar et al., 2012). A similar result has been found in birds, with $M_{\mathrm{b}}$ shown to be negatively correlated with both proton leak (Brand et al., 2003) and ROS generation in liver mitochondria respiring on succinate (Lambert et al., 2007; Montgomery et al., 2012). Collectively, the data suggest (Ramsey et al., 2005; Hagopian et al., 2005), that basal ROS production does not have a negative relationship with basal proton leak. It has previously been reported that ROS generation in the presence of succinate is more sensitive to the $\mathrm{pH}$ gradient than to the proton-motive force or its electrical component (Lambert and Brand, 2004; Lambert et al., 2010). In this study, the low rates of ROS production from the larger frogs were, however, not explained by lower values of $\Delta \mathrm{pH}$ (Table 2).

Another explanation for the difference in ROS production is the difference in the redox state of respiratory chain components. Interestingly, mitochondria from the smallest frogs ( $R$. temporaria) exhibited a lower activity of the substrate oxidation system (Fig. 3A) while having similar non-phosphorylating and phosphorylating oxygen consumption rates to mitochondria from the other frogs. This indicates that mitochondria from the common frog have a low oxidative reserve as illustrated by the lower $\mathrm{fAS}_{\mathrm{m}}$. In turn, this implies that common frog mitochondria operated at a higher proportion of maximal respiration under the phosphorylating state than mitochondria from the other species (i.e. state $3 \mathrm{ADP}$ of R. temporaria, $P$. ridibundus and L. catesbeiana operating at $80 \%$, $75 \%$ and $65 \%$ of FCCP-induced maximal respiration rates, respectively; Table 2). Although we did not directly measure redox potentials of the electron chain carriers, the above result suggests that mitochondria from the small individuals had a more reduced respiration chain while functioning, which would explain the higher ROS production compared with mitochondria from the heavier individuals. Alternatively, because succinate-induced ROS generation is essentially located at complex I (Lambert et al., 2007, 2010), it has also been suggested that the differences in mitochondrial ROS production rates between species can be explained by differences in the number of superoxide production sites within complex I (Lambert et al., 2010). Whether low mitochondrial complex I content and/or low redox potential of the electron carriers may explain differences in mitochondrial ROS production between frog species remains unknown and requires further investigation.

In conclusion, liver mitochondria from frogs show a positive relationship between mitochondrial oxidative phosphorylation efficiency and $M_{\mathrm{b}}$. This is due to a decrease in the mitochondrial proton conductance in the heaviest individuals. Interestingly, the negative relationship found between mitochondrial proton leak and $M_{\mathrm{b}}$ in frogs is similar to that in endothermic groups such as mammals (Porter and Brand, 1993), including marsupials (Polymeropoulos et al., 2012), and birds (Brand et al., 2003). Finally, and in contrast to our prediction, we found that liver mitochondria from the larger individuals produced significantly lower levels of ROS than those from the smaller animals. The molecular mechanism responsible for this negative allometric relationship between mitochondrial ROS production and $M_{\mathrm{b}}$ in frogs remains to be determined. Overall, the heaviest frogs (L. catesbeiana) exhibited a high mitochondrial coupling efficiency and a low level of mitochondrial ROS production, which would drive a high growth rate and promote longevity in this species. The results in this study argue that $M_{\mathrm{b}}$ is dependent on mitochondrial bioenergetics (mitochondrial efficiency, proton leak, ROS production) in frogs, raising the question of whether this would also be the case in other ectothermic groups. Future research should address this using more species before a conclusion can be drawn.

\section{MATERIALS AND METHODS \\ Animals}

Three frog species from the same family (Ranidae) but with differing $M_{\mathrm{b}}$ were used. Common frogs ( $R$. temporaria) were caught on the spawning sites located in Jurassian Bresse between Lons-le-Saunier and Dôle (Jura, France). Marsh frogs ( $P$. ridibundus) were caught near Lyon (Rhône-Alpes, France) and bullfrogs (L. catesbeiana) were caught near Bordeaux (Gironde, France). Animals from the three frog species were maintained individually at $20 \pm 2^{\circ} \mathrm{C}$, with a $12 \mathrm{~h}: 12 \mathrm{~h}$ light:dark cycle and fed on crickets once a week for 2-3 weeks prior to killing by stunning and decapitation. All experiments were carried out according to the ethical principles of the French Ministry of Agriculture, and the French Department of Veterinary Services (DVS no. 69266347).

\section{Mitochondrial isolation}

Individual mitochondrial preparations were from two to five pooled common frog livers, two marsh frog livers, or one bullfrog liver. Liver mitochondria was isolated using differential centrifugation in ice-cold isolation buffer containing $250 \mathrm{mmol}^{-1}$ sucrose, $1 \mathrm{mmol}^{-1}$ EGTA and $20 \mathrm{mmol}^{-1}$ Tris- $\mathrm{HCl}$ brought to $\mathrm{pH} 7.4$ at $4^{\circ} \mathrm{C}$ (Salin et al., 2012b). The protein content of the mitochondrial preparation was assayed at $540 \mathrm{~nm}$ using the biuret method with bovine serum albumin used as a standard. The mitochondrial preparation from frog livers contains a dark pigment which absorbs at $540 \mathrm{~nm}$; therefore, the absorbance of the same volume of mitochondria in isolation buffer containing $0.6 \%$ potassium sodium $\mathrm{L}(+)$-tartrate and $3 \% \mathrm{NaOH}$ was subtracted.

\section{Mitochondrial oxygen consumption and ATP synthesis rates}

Oxygen consumption was measured with a Clark oxygen electrode (Rank Brothers Ltd, France), in a closed and stirred glass cell of $0.5 \mathrm{ml}$ volume and expressed per $\mathrm{mg}$ of mitochondrial protein at $25^{\circ} \mathrm{C}$. Liver mitochondria were incubated in a respiratory buffer containing $120 \mathrm{mmol}^{-1} \mathrm{KCl}, 5 \mathrm{mmol} \mathrm{l}^{-1}$ $\mathrm{KH}_{2} \mathrm{PO}_{4}, 1 \mathrm{mmol} \mathrm{l}^{-1}$ EGTA, 2 mmol $1^{-1} \mathrm{MgCl}_{2}, 0.3 \%$ bovine serum albumin $(\mathrm{w} / \mathrm{v})$ and $3 \mathrm{mmol} \mathrm{1}^{-1}$ Hepes at $\mathrm{pH}$ 7.4. Substrate concentrations used were $5 \mathrm{mmol}^{-1}$ succinate plus $5 \mu \mathrm{mol}^{-1}$ rotenone. The active state of respiration (state $3_{\mathrm{ADP}}$ ) was initiated by the addition of $500 \mu \mathrm{mol} \mathrm{l}^{-1}$ ADP. The basal non-phosphorylating respiration rate $\left(\right.$ state $4_{\text {oligo }}$ ) was measured in the presence of $5 \mu \mathrm{g} \mathrm{ml}^{-1}$ oligomycin. The maximal uncoupled state of respiration (state $3_{\mathrm{FCCP}}$ ) was initiated by the addition of $2 \mu \mathrm{mol} 1^{-1}$ carbonyl cyanide $p$-(trifluoromethoxy)phenylhydrazone (FCCP). The RCR refers to the ratio of ADP-stimulated oxygen consumption (state 3 ) to that consumed in the presence of oligomycin (state $4_{\text {oligo }}$ ). We defined the fractional mitochondrial aerobic scope $\left(\mathrm{fAS}_{\mathrm{m}}\right)$ as the ratio of FCCP-induced maximal uncoupling respiration rate (state $3_{\mathrm{FCCP}}$ ) to oligomycin-induced basal nonphosphorylating respiration rate (state $\left.4_{\text {oligo }}\right)$. These metrics $(\mathrm{RCR}=$ state 3 ADP $/$ state $4_{\text {oligo }}$ or $\mathrm{fAS}_{\mathrm{m}}=$ state $3_{\mathrm{FCCP}} /$ state $4_{\text {oligo }}$ ) provide information on the 
function of oxidative phosphorylation, as state $3_{\mathrm{ADP}}$ is controlled by the activity of ADP phosphorylation and substrate oxidation, state $3_{\mathrm{FCCP}}$ is controlled exclusively by substrate oxidation and state $4_{\text {oligo }}$ is controlled predominantly by the rate at which protons leak across the mitochondrial inner-membrane (Brand and Nicholls, 2011).

Mitochondrial oxidative phosphorylation efficiency was determined by measuring ATP synthesis concurrently with oxygen consumption in respiratory buffer supplemented with $20 \mathrm{mmol}^{-1}$ glucose and $1.5 \mathrm{U} \mathrm{ml}^{-1}$ hexokinase. Different steady states of phosphorylation were obtained by adding ADP from $10 \mu \mathrm{mol} 1^{-1}$ to $100 \mu \mathrm{mol} 1^{-1}$. Mitochondrial ATP synthesis was determined from the glucose 6-phosphate content of the samples, which was measured enzymatically by spectrophotometry as previously described (Salin et al., 2012b). Briefly, after recording the respiration rate, four aliquots ( $60 \mu \mathrm{l}$ each) of mitochondrial suspension were withdrawn from the respiratory chamber every $2 \mathrm{~min}$ and immediately added to $40 \mu$ of perchloric acid solution $\left(10 \% \mathrm{HClO}_{4}, 25 \mathrm{mmol} \mathrm{l}^{-1}\right.$ EDTA $)$ to stop the reaction. After centrifugation of the denatured protein $(15,000 \mathrm{~g}$ for $5 \mathrm{~min}$ ) and neutralization of the resulting supernatant, the glucose 6-phosphate content of the samples was measured by spectrophotometry at $340 \mathrm{~nm}$ in an assay medium consisting of $50 \mathrm{mmol} \mathrm{l}^{-1}$ tri-ethanolamine$\mathrm{HCl}, 7.5 \mathrm{mmol}^{-1} \mathrm{MgCl}_{2}, 3.75 \mathrm{mmol}^{-1}$ EDTA, $0.5 \mathrm{mmol}^{-1} \mathrm{NAD}$ and $0.5 \mathrm{U}$ glucose 6-phosphate dehydrogenase, $\mathrm{pH}$ 7.4. The rate of mitochondrial ATP production was calculated from the slope of the linear accumulation of glucose 6-phosphate over the sampling time interval (6 min). The linearity of glucose 6-phosphate accumulation was checked to ensure that the system was in a steady state. We also ensured that the ATP production measured was specific to mitochondrial ATP synthase activity by determining oxygen consumption and ATP synthesis rates in the presence of oligomycin $\left(5 \mu \mathrm{g} \mathrm{ml}^{-1}\right)$. Over the range of ADP concentrations used, no oligomycin-insensitive ATP synthesis activity was measurable.

\section{Mitochondrial membrane potential}

Respiration rate and membrane potential were measured simultaneously using electrodes sensitive to oxygen and to the potential-dependent probe triphenylmethylphosphonium (TPMP ${ }^{+}$) (Brand, 1995; Brookes et al., 1998; Salin et al., 2012b). Mitochondria were incubated in respiratory buffer supplemented with $5 \mu \mathrm{g} \mathrm{ml}^{-1}$ oligomycin. The $\mathrm{TPMP}^{+}$electrode was calibrated with four sequential $0.5 \mu \mathrm{mol} 1^{-1}$ additions up to $2 \mu \mathrm{mol} 1^{-1}$ $\mathrm{TPMP}^{+}$, and then $5 \mathrm{mmol}^{-1}$ succinate was added to start the reaction and membrane potential was measured upon reaching the steady state. The chemical component of proton-motive force, $\Delta \mathrm{pH}$, was then measured as the change in membrane potential after $\Delta \mathrm{pH}$ was converted to the electrical component of proton-motive force $(\Delta \psi)$ by addition of $60 \mathrm{ng} \mathrm{ml}^{-1}$ nigericin (Brand, 1995). On the basis that proton-motive force $=\Delta \psi+\Delta \mathrm{pH}, \Delta \mathrm{pH}$ is simply calculated as $\Delta \mathrm{pH}=$ proton-motive force ( presence of nigericin) $-\Delta \psi$ (absence of nigericin) (Lambert and Brand, 2004). The kinetic response of proton leak and substrate oxidation to proton-motive force was determined by titration of non-phosphorylating respiration with malonate (up to $5 \mathrm{mmol} \mathrm{l}^{-1}$ ) or FCCP (up to $800 \mathrm{nmol}^{-1}$ ), respectively (Brand and Nicholls, 2011). After each run, $2 \mu \mathrm{mol}^{-1}$ FCCP was added to dissipate the membrane potential and release all $\mathrm{TPMP}^{+}$back into the medium for baseline correction. Membrane potential was calculated as described by Brand (1995), assuming a TPMP binding correction of $0.69 \mathrm{mg}$ protein $\mu^{-1}$ for liver mitochondria (Brand et al., 1991; Brookes et al., 1998). For $P$. ridibundus and $L$. catesbeiana, mitochondrial oxygen consumption and membrane potential were assayed at $25^{\circ} \mathrm{C}$. For $R$. temporaria, mitochondrial oxygen consumption and membrane potential were assayed at $20^{\circ} \mathrm{C}$ (values are from Salin et al., 2012b) and corresponding values were recalculated to $25^{\circ} \mathrm{C}$ assuming a $Q_{10}$ of 2.3 for oxygen consumption and of 1.05 for membrane potential (Berner, 1999; Chamberlin, 2004; Rogers et al., 2007; Trzcionka et al., 2008; Guderley and Seebacher, 2011). For all three species, the rates of mitochondrial proton leak were calculated at the highest common membrane potential value of $150 \mathrm{mV}$ from the oxygen consumption rate by assuming a constant stoichiometry of six protons pumped (and leaked) per atom of oxygen for succinate. It is important to note that one previous study has reported a proton leak activity of around $30 \mathrm{nmol} \mathrm{H}^{+} \mathrm{min}^{-1} \mathrm{mg}^{-1}$ protein in skeletal muscle mitochondria of common frog at $25^{\circ} \mathrm{C}$ (St-Pierre et al., 2000). Given that basal non-phosphorylating respiration rate and basal proton leak activity are on average 3.4- or 2.5-fold greater in skeletal muscle mitochondria than in liver mitochondria, respectively (St-Pierre et al., 2000; Hulbert et al., 2006; Rogers et al., 2007; Trzcionka et al., 2008; Guderley and Seebacher, 2011), the proton leak activity value of liver mitochondria from common frog should have ranged from 14 to $8.7 \mathrm{nmol} \mathrm{H}^{+} \mathrm{min}^{-1} \mathrm{mg}^{-1}$ protein (at $150 \mathrm{mV}$ and $25^{\circ} \mathrm{C}$ ). Hence, in the present study, the calculated mean value of $9.7 \pm 1.2 \mathrm{nmol} \mathrm{H}^{+} \mathrm{min}^{-1} \mathrm{mg}^{-1}$ protein (Fig. 3B) is in the range of expected values, showing that the above $Q_{10}$ values are reasonable.

\section{Mitochondrial ROS production and liver superoxide dismutase activity}

The rate of $\mathrm{H}_{2} \mathrm{O}_{2}$ released from mitochondria was measured using a fluorescence spectrophotometer (Xenius, SAFAS Monaco) at excitation and emission wavelengths of 563 and $587 \mathrm{~nm}$, respectively. The respiratory buffer was supplemented with $5 \mathrm{U} \mathrm{ml}^{-1}$ horseradish peroxidase and $1 \mu \mathrm{mol} 1^{-1}$ Amplex red fluorescent dye. Mitochondria and succinate were added to start the reaction. The fluorescent signal was calibrated using a standard curve obtained after successive addition of $\mathrm{H}_{2} \mathrm{O}_{2} \quad(20$ $400 \mathrm{nmol}^{-1}$ ). Electron leak was calculated as the fraction (\%) of total electron flow that reduces $\mathrm{O}_{2}$ into oxygen free radicals at the respiratory chain instead of reaching cytochrome $c$ oxidase to reduce $\mathrm{O}_{2}$ into $\mathrm{H}_{2} \mathrm{O}$. The total activity of liver superoxide dismutase was determined as previously described (Rey et al., 2010).

\section{Statistical analyses}

Results are presented as means \pm s.e.m. An ANCOVA was performed to test, for every parameter, both species and individual mass effects together with their interactions. Data were log transformed to homogenize variances when homoscedasticity was not observed. Statistical analyses were performed using JMP 7 (SAS Institute Inc., Cary, NC, USA). A 5\% $(P=0.05)$ level of significance was used in all tests.

\section{Acknowledgements}

We are grateful to Amy Sinclair for help with the English and to the reviewers for their helpful comments.

\section{Competing interests}

The authors declare no competing or financial interests.

\section{Author contributions}

D.R. conceived and conducted the bioenergetics studies, performed the mitochondrial membrane potential and proton conductance experiments, analyzed the data, assembled the figures and wrote de manuscript. K.S. performed the bioenergetics and ROS production experiments, analyzed the data and revised the article. A.D. and C.R. performed the enzymatic analyses. B.R. performed the ROS production analyses and revised the article. Y.V. conceived and designed the study, analyzed the data and revised the article.

\section{Funding}

K.S. was in receipt of a fellowship from the French Ministère de l'Enseignement Supérieur et de la Recherche.

\section{References}

Akhmerov, R. N. (1986). Qualitative difference in mitochondria of endothermic and ectothermic animals. FEBS Lett. 198, 251-255.

Berner, N. J. (1999). Oxygen consumption by mitochondria from an endotherm and an ectotherm. Comp. Biochem. Physiol. B Biochem. Mol. Biol. 124, 25-31.

Berner, N. J., Else, P. L., Hulbert, A. J., Mantle, B. L., Cramp, R. L. and Franklin, C. E. (2009). Metabolic depression during aestivation does not involve remodelling of membrane fatty acids in two Australian frogs. J. Comp. Physiol. B 179, 857-866.

Bottje, W., Brand, M. D., Ojano-Dirain, C., Lassiter, K., Toyomizu, M. and Wing, T. (1999). Mitochondrial proton leak kinetics and relationship with feed efficiency within a single genetic line of male broilers. Poult. Sci. 88, 1683-1693.

Brand, M. D. (1995). Bioenergetics, a Practical Approach (ed. Brown G.C. and Cooper C.E.), pp. 39-62. Oxford: IRL Press.

Brand, M. D. (2000). Uncoupling to survive? The role of mitochondrial inefficiency in ageing. Exp. Gerontol. 35, 811-820.

Brand, M. D. (2005). The efficiency and plasticity of mitochondrial energy transduction. Biochem. Soc. Trans. 33, 897-904. 
Brand, M. D. and Nicholls, D. G. (2011). Assessing mitochondrial dysfunction in cells. Biochem. J. 435, 297-312.

Brand, M. D., Couture, P., Else, P. L., Withers, K. W. and Hulbert, A. J. (1991) Evolution of energy metabolism. Proton permeability of the inner membrane of liver mitochondria is greater in a mammal than in a reptile. Biochem. J. 275, 81-86.

Brand, M. D., Turner, N., Ocloo, A., Else, P. L. and Hulbert, A. J. (2003). Proton conductance and fatty acyl composition of liver mitochondria correlates with body mass in birds. Biochem. J. 376, 741-748.

Brand, M. D., Pakay, J. L., Ocloo, A., Kokoszka, J., Wallace, D. C., Brookes, P. S. and Cornwall, E. J. (2005). The basal proton conductance of mitochondria depends on adenine nucleotide translocase content. Biochem. J.392, 353-362.

Brookes, P. S., Hulbert, A. J. and Brand, M. D. (1997). The proton permeability of liposomes made from mitochondrial inner membrane phospholipids: no effect of fatty acid composition. Biochim. Biophys. Acta 1330, 157-164.

Brookes, P. S., Buckingham, J. A., Tenreiro, A. M., Hulbert, A. J. and Brand, M. D. (1998). The proton permeability of the inner membrane of liver mitochondria from ectothermic and endothermic vertebrates and from obese rats: correlations with standard metabolic rate and phospholipid fatty acid composition. Comp. Biochem. Physiol. 119, 325-334.

Caldeira da Silva, C. C., Cerqueira, F. M., Barbosa, L. F., Medeiros, M. H. G. and Kowaltowski, A. J. (2008). Mild mitochondrial uncoupling in mice affects energy metabolism, redox balance and longevity. Aging Cell 7, 552-560.

Chamberlin, M. E. (2004). Top-down control analysis of the effect of temperature on ectotherm oxidative phosphorylation. Am. J. Physiol. 287, R794-R800.

Csiszar, A., Podlutsky, A., Podlutskaya, N., Sonntag, W. E., Merlin, S. Z., Philipp, E. E. R., Doyle, K., Davila, A., Recchia, F. A., Ballabh, P. et al. (2012). Testing the oxidative stress hypothesis of aging in primate fibroblasts: is there a correlation between species longevity and cellular ROS production? J. Gerontol. A Biol. Sci. Med. Sci. 67, 841-852.

Darveau, C.-A., Suarez, R. K., Andrews, R. D. and Hochachka, P. W. (2002) Allometric cascade as a unifying principle of body mass effects on metabolism. Nature 417, 166-170

Else, P. L. and Hulbert, A. J. (1985). An allometric comparison of the mitochondria of mammalian and reptilian tissues: the implications for the evolution of endothermy. J. Comp. Physiol. B 156, 3-11.

Else, P. L., Brand, M. D., Turner, N. and Hulbert, A. J. (2004). Respiration rate of hepatocytes varies with body mass in birds. J. Exp. Biol. 207, 2305-2311.

Glazier, D. S. (2005). Beyond the "3/4-power law": variation in the intra- and interspecific scaling of metabolic rate in animals. Biol. Rev. 80, 611-662.

Guderley, H. and Seebacher, F. (2011). Thermal acclimation, mitochondria capacities and organ metabolic profiles in a reptile (Alligator mississippiensis) J. Comp. Physiol. B 181, 53-64.

Hagopian, K., Harper, M. E., Ram, J. J., Humble, S. J., Weindruch, R. and Ramsey, J. J. (2005). Long-term calorie restriction reduces proton leak and hydrogen peroxide production in liver mitochondria. Am. J. Physiol. 288, E674-E684.

Hulbert, A. J. and Else, P. L. (2000). Mechanisms underlying the cost of living in animals. Annu. Rev. Physiol. 62, 207-235

Hulbert, A., Else, P., Manolis, S. and Brand, M. (2002). Proton leak in hepatocytes and liver mitochondria from archosaurs (crocodiles) and allometric relationships for ectotherms. J. Comp. Physiol. B Biochem. Syst. Environ. Physiol. 172 387-397.

Hulbert, A. J., Turner, N., Hinde, J., Else, P. and Guderley, H. (2006). How migh you compare mitochondria from different tissues and different species? J. Comp. Biochem. B 176, 93-105.

Kikusato, M. and Toyomizu, M. (2013). Crucial role of membrane potential in heat stress-induced overproduction of reactive oxygen species in avian skeletal muscle mitochondria. PLOS ONE 8, e64412.

Korshunov, S. S., Skulachev, V. P. and Starkov, A. A. (1997). High protonic potential actuates a mechanism of production of reactive oxygen species in mitochondria. FEBS Lett. 416, 15-18.

Ku, H.-H., Brunk, U. T. and Sohal, R. S. (1993). Relationship between mitochondrial superoxide and hydrogen peroxide production and longevity of mammalian species. Free Radic. Biol. Med. 15, 621-627.

Kunkel, H. O. and Campbell, J. E., Jr. (1952). Tissue cytochrome oxidase activity and body weight. J. Biol. Chem. 198, 229-236.

Lambert, A. J. and Brand, M. D. (2004). Superoxide production by NADH: ubiquinone oxidoreductase (complex I) depends on the $\mathrm{pH}$ gradient across the mitochondrial inner membrane. Biochem. J. 382, 511-517.

Lambert, A. J., Boysen, H. M., Buckingham, J. A., Yang, T., Podlutsky, A., Austad, S. N., Kunz, T. H., Buffenstein, R. and Brand, M. D. (2007). Low rates of hydrogen peroxide production by isolated heart mitochondria associate with long maximum lifespan in vertebrate homeotherms. Aging Cell 6, 607-618.

Lambert, A. J., Buckingham, J. A., Boysen, H. M. and Brand, M. D. (2010). Low complex I content explains the low hydrogen peroxide production rate of heart mitochondria from the long-lived pigeon, Columba livia. Aging Cell 9, 78-91.

Miwa, S., St-Pierre, J., Partridge, L. and Brand, M. (2003). Superoxide and hydrogen peroxide production by Drosophila mitochondria. Free Radic. Biol. Med. 35, 938-948.

Montgomery, M. D., Hulbert, A. J. and Buttemer, W. A. (2012). Does the oxidative stress theory of aging explain longevity differences in birds? I. Mitochondrial ROS production. Exp. Gerontol. 47, 203-210.

Mookerjee, S. A., Divakaruni, A. S., Jastroch, M. and Brand, M. D. (2010) Mitochondrial uncoupling and lifespan. Mech. Ageing Dev. 131, 463-472.

Nagy, K. A. (2005). Field metabolic rate and body size. J. Exp. Biol. 208, 1621-1625

Polymeropoulos, E. T., Heldmaier, G., Frappell, P. B., McAllan, B. M., Withers K. W., Klingenspor, M., White, C. R. and Jastroch, M. (2012). Phylogenetic differences of mammalian basal metabolic rate are not explained by mitochondrial basal proton leak. Proc. R. Soc. B Biol. Sci. 279, 185-193.

Porter, R. K. and Brand, M. D. (1993). Body mass dependence of $\mathrm{H}^{+}$leak in mitochondria and its relevance to metabolic rate. Nature $362,628-630$.

Porter, R. K., Hulbert, A. J. and Brand, M. D. (1996). Allometry of mitochondria proton leak: influence of membrane surface area and fatty acid composition. Am. J. Physiol. 271, R1550-R1560

Ramsey, J. J., Harper, M.-E., Humble, S. J., Koomson, E. K., Ram, J. J. Bevilacqua, L. and Hagopian, K. (2005). Influence of mitochondrial membrane fatty acid composition on proton leak and $\mathrm{H} 2 \mathrm{O} 2$ production in liver. Comp. Biochem. Physiol. B Biochem. Mol. Biol. 140, 99-108.

Rey, B., Roussel, D., Romestaing, C., Belouze, M., Rouanet, J.-L., Desplanches D., Sibille, B., Servais, S. and Duchamp, C. (2010). Up-regulation of avian uncoupling protein in cold-acclimated and hyperthyroid ducklings prevents reactive oxygen species production by skeletal muscle mitochondria. BMC Physiol. 10, 5

Rogers, K. D., Thompson, M. B. and Seebacher, F. (2007). Beneficial acclimation: sex specific thermal acclimation of metabolic capacity in the striped marsh frog (Limnodynastes peronii). J. Exp. Biol. 210, 2932-2938.

Salin, K., Luquet, E., Rey, B., Roussel, D. and Voituron, Y. (2012a). Alteration of mitochondrial efficiency affects oxidative balance, development and growth in frog (Rana temporaria) tadpoles. J. Exp. Biol. 215, 863-869.

Salin, K., Roussel, D., Rey, B. and Voituron, Y. (2012b). David and Goliath: a mitochondrial coupling problem? J. Exp. Zool. 317, 283-293.

Savina, M. V., Emelyanova, L. V. and Belyaeva, E. A. (2006). Bioenergetic parameters of lamprey and frog liver mitochondria during metabolic depression and activity. Comp. Biochem. Physiol. B Biochem. Mol. Biol. 145, 296-305.

Schmidt-Nielsen, K. (1984). Scaling: Why is Animal Size so Important? New York: Cambridge University Press.

Shabalina, I. G., Kramarova, T. V., Nedergaard, J. and Cannon, B. (2006) Carboxyatractyloside effects on brown-fat mitochondria imply that the adenine nucleotide translocator isoforms ANT1 and ANT2 may be responsible for basal and fatty-acid-induced uncoupling respectively. Biochem. J. 399, 405-414

Sohal, R. S., Svensson, I., Sohal, B. H. and Brunk, U. T. (1989). Superoxide anion radical production in different animal species. Mech. Ageing Dev. 49, 129-135.

Sohal, R. S., Svensson, I. and Brunk, U. T. (1990). Hydrogen peroxide production by liver mitochondria in different species. Mech. Ageing Dev. 53, 209-215.

St-Pierre, J., Brand, M. D. and Boutilier, R. G. (2000). The effect of metabolic depression on proton leak rate in mitochondria from hibernating frogs. J. Exp. Biol. 203, 1469-1476.

Stuart, J. A., Cadenas, S., Jekabsons, M. B., Roussel, D. and Brand, M. D. (2001). Mitochondrial proton leak and the uncoupling protein 1 homologues. Biochim. Biophys. Acta 1504, 144-158.

Talbot, D. A., Duchamp, C., Rey, B., Hanuise, N., Rouanet, J. L., Sibille, B. and Brand, M. D. (2004). Uncoupling protein and ATP/ADP carrier increase mitochondrial proton conductance after cold adaptation of king penguins. J. Physiol. 558, 123-135

Toyomizu, M., Okamoto, K., Tanaka, M. and Ishibashi, T. (1992). Effect of 2,4dinitrophenol on growth and body composition of broilers. Poult. Sci. 71 1096-1100.

Trzcionka, M., Withers, K. W., Klingenspor, M. and Jastroch, M. (2008). The effects of fasting and cold exposure on metabolic rate and mitochondrial proton leak in liver and skeletal muscle of an amphibian, the cane toad Bufo marinus. J. Exp. Biol. 211, 1911-1918. 